\title{
Study of Monitoring Works Using Artificial Neural Network
}

\author{
Zhiming Guo ${ }^{1}$, Xiangyang Chen ${ }^{2}$, Minghui Tang ${ }^{1}$, Weikai Liu ${ }^{2}$ and Min Zhang ${ }^{2}$ \\ ${ }^{1}$ Faculty of Engineering China University of Geosciences Wuhan, Hubei Province, China \\ ${ }^{2}$ School of Computer Science and Engineering Wuhan Institute of Technology, Wuhan, Hubei Province, China \\ xychensun@yahoo.com.cn
}

\begin{abstract}
As a giant and complicated project, the construction of Shibaozhai Breakwater in Chongqing needs subtle prediction of its stability and reliability. Such work is with great difficulty. However, by using artificial neural network under the MATLAB,we use the measured dataset to model the stability and reliability of the Breakwater project thus to forecast the index concerned.
\end{abstract}

Index Terms - Artificial neural network; MATLAB; Breakwater; Application Research.

\section{Introduction}

With huge investment, the construction of Chongqing Shibaozhai Breakwater project matters the security of Shibaozhai. Therefore it significant to anticipate its stability and reliability with precision. The measured data are collected in the process of construction by BGK steel stress meter and Inclinometer. With rapid development of artificial neural network theory, its application in structure reliability analysis gradually attains great focus. Artificial neural network is a nonlinear system made up of enormous processing units, which enjoys lots of advantages like: flexible adaptability, self- organization, quick learning ability, fault-tolerance, and anti-disturbance ability. It also can create efficient model directly based on sampled data[1] by avoid the complicated mechanism of an unknown system.

However, the realization of the network model needs high proficiency of the programmer and researcher, which, to some extent, hinders the coverage and promotion of artificial neural network. To solve this problem, the use of MATLAB neural network toolkit is introduced to saves certain programming and debugging time. Thus the research can be concentrated on the modeling of the urgent problem, with great improvement in efficiency.[2]

\section{Basic Principal And Procedure}

The following are the analyses of measured data. We preprocess on the original data, use command premnmx(p) normalize data $\mathrm{p}(-1,1)$, then set up initial network, and train the network with data. Here we adopted Levenberg- $M$ arquardt Algorithm, because it is very fast for the middle scale BP neural network, and Convergence error is small. Other network parameter set as: net.trainparam.epochs $=2000$; net.trainparam.goal $=0.001$; net.trainparam.show $=20$; net.trainparam.lr $=0.6$; net.trainparam. $m c=0.6$. Finally we use well-trained neural network, according to the time series, to proceed 20 times Monte Carlo simulation, and anticipate the future 5 steps[3].

TABLE 1 horizontal displacement of the first Random selected sectional observation

\begin{tabular}{|c|c|c|c|c|c|c|}
\hline Date elevation $(\mathrm{m})$ & 136.7 & 138.2 & 142.2 & 147.7 & 151.7 & 153.2 \\
\hline $2006-9-4$ & 0.00 & 0.01 & 0.00 & 0.00 & 0.00 & 0.00 \\
$2006-9-10$ & 0.55 & 0.68 & 0.18 & 0.16 & 0.36 & 0.10 \\
$2006-9-18$ & 0.62 & 0.73 & 0.75 & 0.23 & 0.43 & 0.23 \\
$2006-9-23$ & 0.77 & 0.61 & 0.60 & 0.36 & 0.10 & 0.00 \\
$2006-9-27$ & 0.70 & 0.70 & 0.40 & 0.43 & 0.18 & 0.18 \\
$2006-10-2$ & 0.64 & 0.91 & 0.45 & 0.48 & 0.33 & 0.30 \\
$2006-10-7$ & 0.67 & 0.90 & 0.53 & 0.43 & 0.16 & 0.38 \\
$2006-10-12$ & 0.57 & 1.03 & 1.00 & 0.38 & 0.36 & 0.35 \\
$2006-10-16$ & 0.32 & 0.93 & 0.65 & 0.28 & 0.63 & 0.40 \\
$2006-11-5$ & 0.67 & -0.27 & -3.90 & 0.73 & 0.38 & 1.75 \\
$2007-8-8$ & 0.20 & 0.03 & -0.12 & 0.78 & 1.23 & 1.28 \\
$2007-8-21$ & 0.57 & 0.26 & 0.00 & 0.86 & 1.55 & 1.28 \\
$2007-9-8$ & 0.42 & 0.06 & -0.17 & 0.58 & 1.26 & 1.15 \\
$2007-9-17$ & -1.38 & -2.12 & -2.75 & -1.97 & -1.77 & -1.85 \\
$2007-9-24$ & 0.64 & 0.33 & -0.05 & 1.08 & 1.55 & 1.40 \\
$2007-10-3$ & 0.37 & -0.15 & -0.13 & 0.83 & 1.40 & 1.30 \\
$2007-10-11$ & 0.04 & -0.20 & 0.28 & 0.23 & 0.88 & 0.83 \\
$2008-3-2$ & 0.17 & -0.02 & -1.35 & 0.68 & 1.23 & 2.90 \\
$2008-3-5$ & 0.02 & -0.22 & -1.10 & 0.63 & 1.03 & 2.70 \\
\hline
\end{tabular}

\section{Elevation $136.7 \mathrm{~m}$}

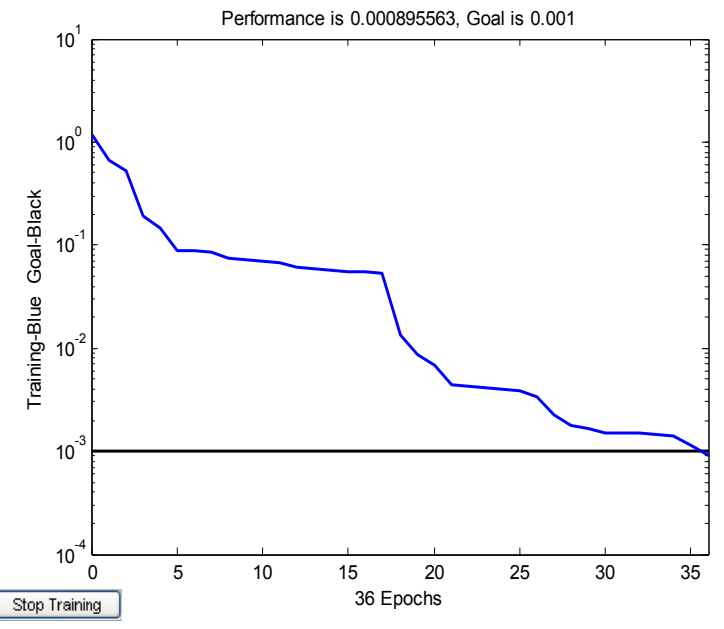

Fig. 1 training steps and performance 


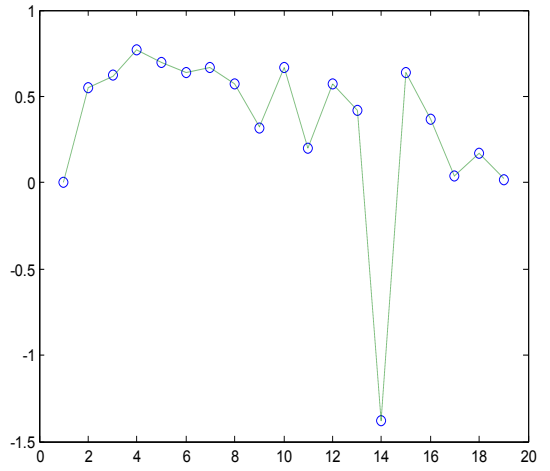

Fig. 2 horizontal displacement data(Dotted line) prediction data(mark with circles)

TABLE 2 The error of the prediction result analysis

\begin{tabular}{|l|c|c|c|}
\hline \multicolumn{1}{|c|}{ Date } & Actual data & Prediction data & absolute errors \\
\hline $2007-8-8$ & 0.20 & 0.2048 & 0.0048 \\
$2007-8-21$ & 0.57 & 0.5691 & -0.0009 \\
$2007-9-8$ & 0.42 & 0.4242 & 0.0042 \\
$2007-9-17$ & -1.38 & -1.3728 & 0.0072 \\
$2007-9-24$ & 0.64 & 0.6302 & -0.0098 \\
$2007-10-3$ & 0.37 & 0.3763 & 0.0063 \\
$2007-10-11$ & 0.04 & 0.0284 & -0.0116 \\
$2008-3-2$ & 0.17 & 0.1688 & -0.0012 \\
$2008-3-5$ & 0.02 & 0.0338 & 0.0138 \\
\hline
\end{tabular}

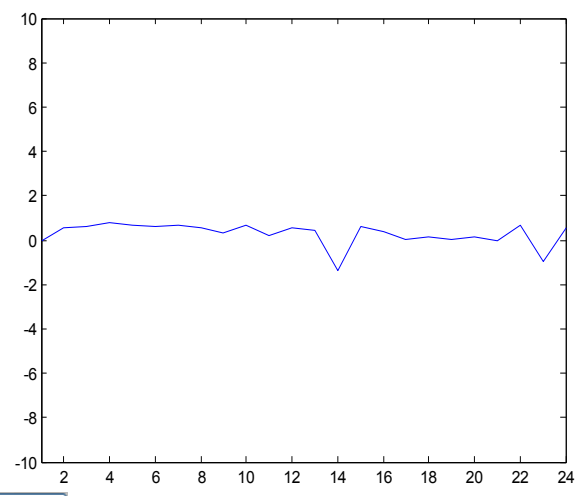

Stop Training

Fig. 3 Elevation $136.7 \mathrm{~m}$ measured data and 5 steps prediction curve

\section{Elevation $138.2 \mathrm{~m}$}

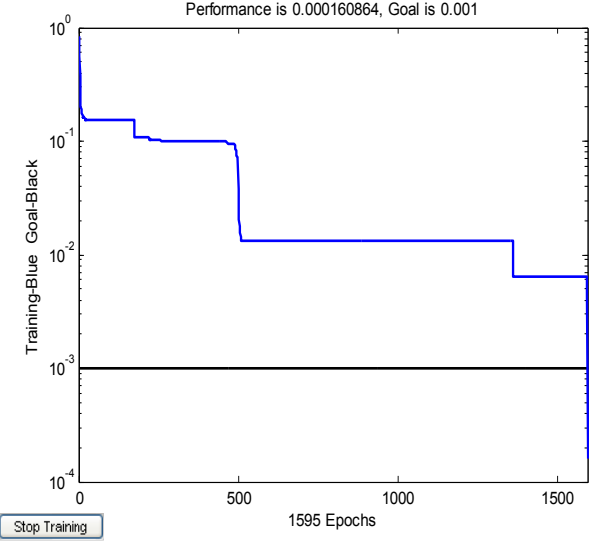

Fig.4 training steps and performance

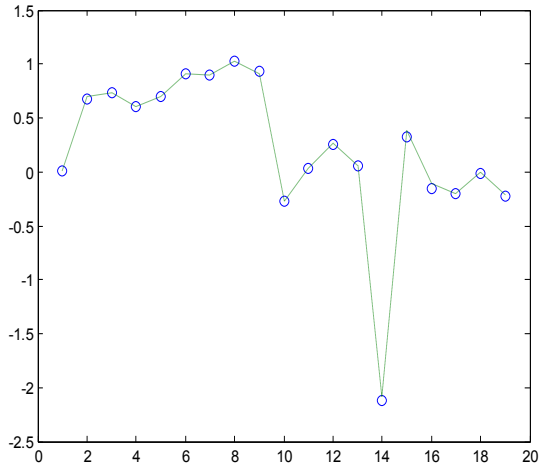

Fig.5 horizontal displacement data(Dotted line) prediction data(mark with circles)

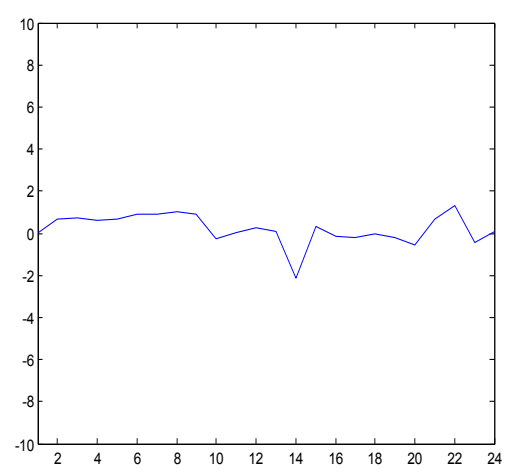

Fig.6 Elevation $138.2 \mathrm{~m}$ measured data and 5 steps prediction curve

TABLE 3 The error of the prediction result analysis

\begin{tabular}{|l|c|c|c|}
\hline \multicolumn{1}{|c|}{ Date } & Actual data & Prediction data & Absolute errors \\
\hline $2007-8-8$ & 0.03 & 0.0286 & -0.0014 \\
$2007-8-21$ & 0.26 & 0.2635 & 0.0035 \\
$2007-9-8$ & 0.06 & 0.0547 & -0.0053 \\
$2007-9-17$ & -2.12 & -2.1160 & 0.0040 \\
$2007-9-24$ & 0.33 & 0.3238 & -0.0062 \\
$2007-10-3$ & -0.15 & -0.1092 & 0.0408 \\
$2007-10-11$ & -0.20 & -0.1790 & 0.0210 \\
$2008-3-2$ & -0.02 & 0.0008 & 0.0208 \\
$2008-3-5$ & -0.22 & -0.1593 & 0.0607 \\
\hline
\end{tabular}

\section{Elevation $142.2 m$ :}

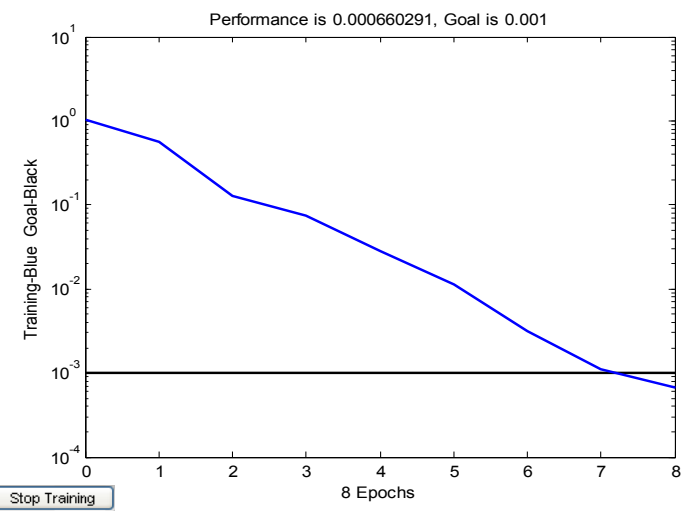

Fig.7 training steps and performance 


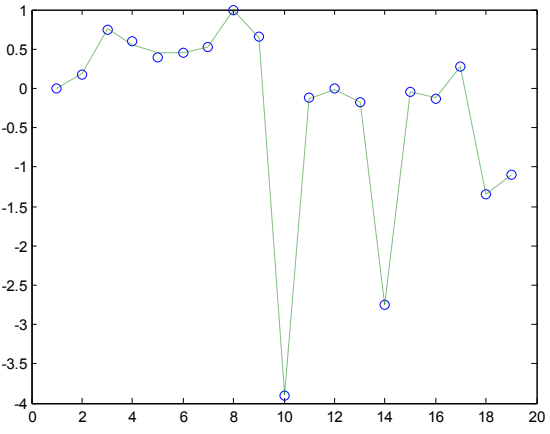

Fig.8 horizontal displacement data(Dotted line $)$ prediction data(mark with circles)

TABLE 4 The error of the prediction result analysis

\begin{tabular}{|l|c|c|c|}
\hline \multicolumn{1}{|c|}{ Date } & Actual data & prediction data & absolute errors \\
\hline $2007-8-8$ & -0.12 & -0.1249 & -0.0049 \\
$2007-8-21$ & 0.00 & -0.0041 & -0.0041 \\
$2007-9-8$ & -0.17 & -0.1633 & 0.0067 \\
$2007-9-17$ & -2.75 & -2.7493 & 0.0007 \\
$2007-9-24$ & -0.05 & -0.0546 & -0.0046 \\
$2007-10-3$ & -0.13 & -0.1473 & -0.0173 \\
$2007-10-11$ & 0.28 & 0.2724 & -0.0076 \\
$2008-3-2$ & -1.35 & -1.3469 & 0.0031 \\
$2008-3-5$ & -1.10 & -1.0961 & 0.0039 \\
\hline
\end{tabular}

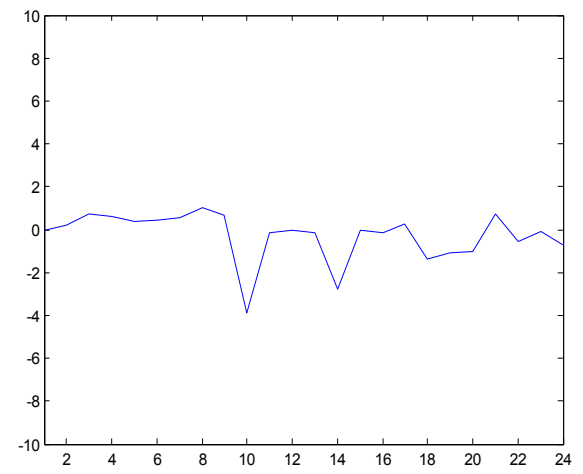

Fig.9 Elevation $142.2 \mathrm{~m}$ measured data and 5 steps prediction curve

\section{Elevation $147.7 m$ :}

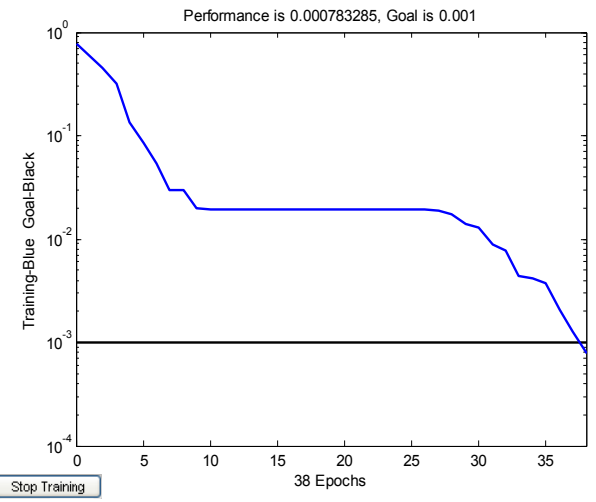

Fig.10 training steps and performance

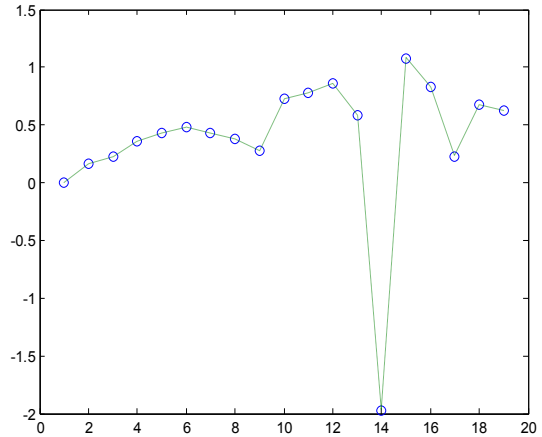

Fig.11 horizontal displacement data(Dotted line) prediction data(mark with circles)

TABLE 5 The error of the prediction result analysis

\begin{tabular}{|c|c|c|c|}
\hline Date & Actual data & prediction data & absolute errors \\
\hline $2007-8-8$ & 0.78 & 0.7794 & -0.0006 \\
$2007-8-21$ & 0.86 & 0.8597 & -0.0003 \\
$2007-9-8$ & 0.58 & 0.5796 & -0.0004 \\
$2007-9-17$ & -1.97 & -1.9718 & -0.0018 \\
$2007-9-24$ & 1.08 & 1.0747 & -0.0053 \\
$2007-10-3$ & 0.83 & 0.8327 & 0.0027 \\
$2007-10-11$ & 0.23 & 0.2348 & 0.0048 \\
$2008-3-2$ & 0.68 & 0.6763 & -0.0037 \\
$2008-3-5$ & 0.63 & 0.6268 & -0.0032 \\
\hline
\end{tabular}

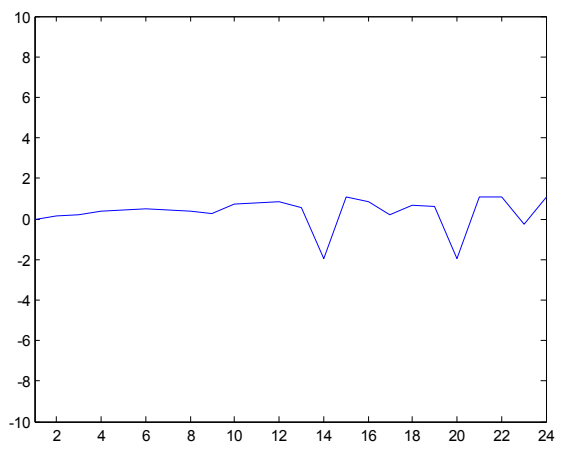

Fig.12 Elevation $147.7 \mathrm{~m}$ measured data and 5 steps prediction curve

\section{Elevation $151.7 m$ :}

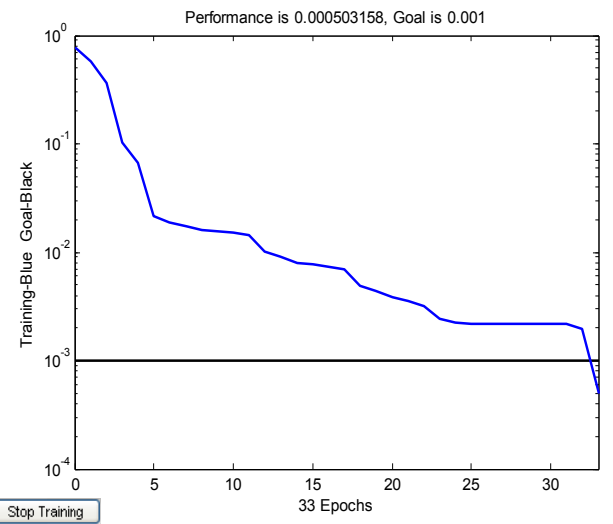

Fig.13 training steps and performance 


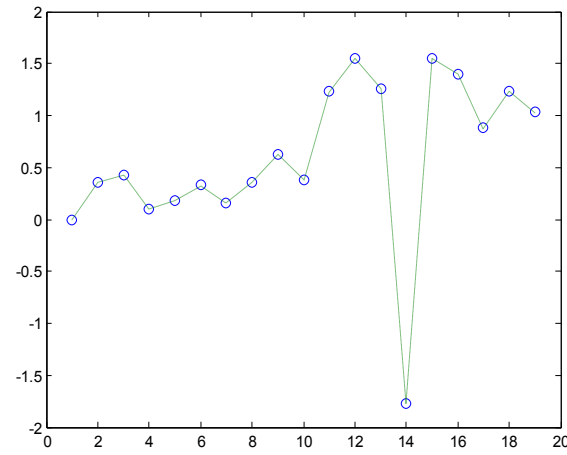

Fig.14 horizontal displacement data(Dotted line) prediction data(mark with circles)

TABLE 6 The error of the prediction result analysis

\begin{tabular}{|l|c|c|c|}
\hline \multicolumn{1}{|c|}{ Date } & Actual data & prediction data & absolute errors \\
\hline $2007-8-8$ & 1.23 & 1.2304 & 0.0004 \\
$2007-8-21$ & 1.55 & 1.5484 & -0.0016 \\
$2007-9-8$ & 1.26 & 1.2592 & -0.0008 \\
$2007-9-17$ & -1.77 & -1.7655 & 0.0045 \\
$2007-9-24$ & 1.55 & 1.5543 & 0.0043 \\
$2007-10-3$ & 1.40 & 1.3986 & -0.0014 \\
$2007-10-11$ & 0.88 & 0.8814 & 0.0014 \\
$2008-3-2$ & 1.23 & 1.2288 & -0.0012 \\
$2008-3-5$ & 1.03 & 1.0256 & -0.0044 \\
\hline
\end{tabular}

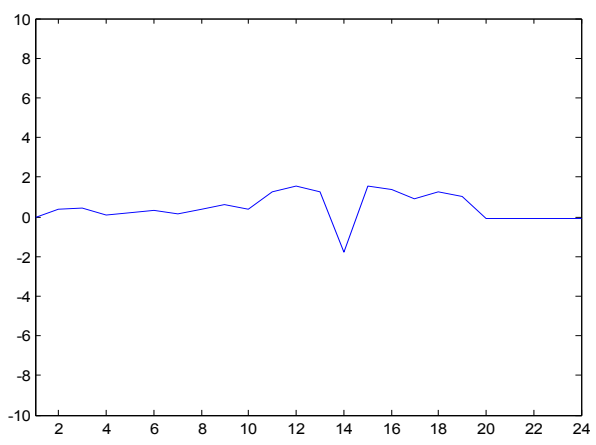

Fig. 15 Elevation $151.7 \mathrm{~m}$ measured data and 5 steps prediction curve

\section{Elevation $153.2 \mathrm{~m}$ :}

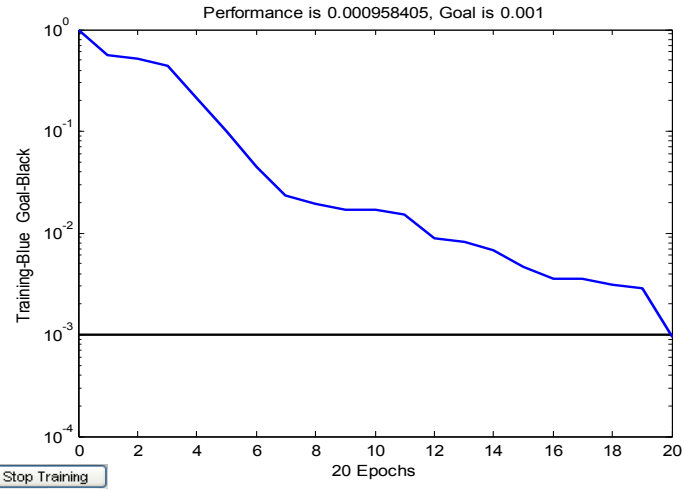

Fig.16 training steps and performance

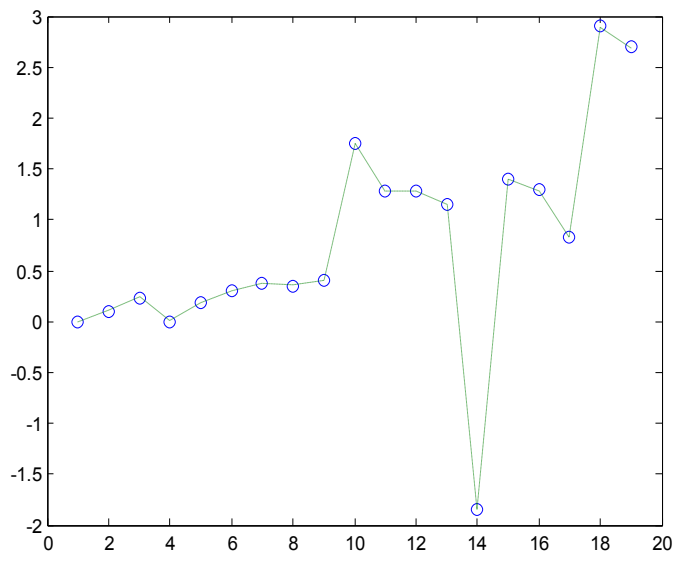

Fig. 17 horizontal displacement data(Dotted line) prediction data(mark with circles)

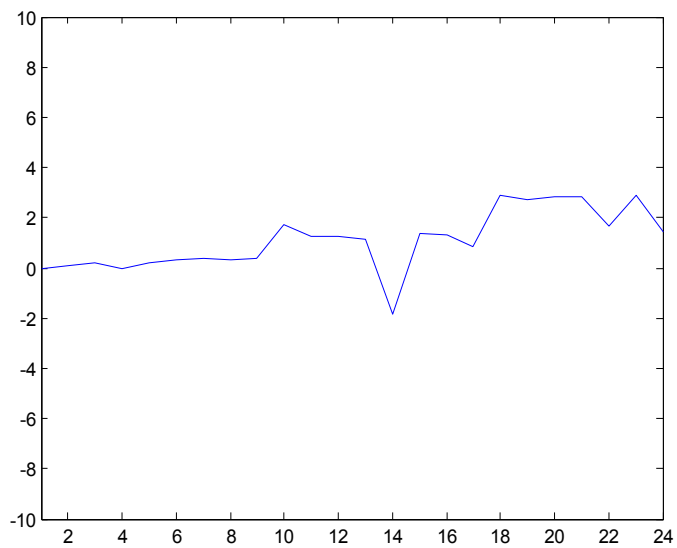

Fig.18 Elevation $153.2 \mathrm{~m}$ measured data and 5 steps prediction curve

TABLE 7 The error of the prediction result analysis

\begin{tabular}{|l|c|c|c|}
\hline \multicolumn{1}{|c|}{ Date } & Actual data & prediction data & absolute errors \\
\hline $2007-8-8$ & 1.28 & 1.2804 & 0.0004 \\
$2007-8-21$ & 1.28 & 1.2830 & 0.003 \\
$2007-9-8$ & 1.15 & 1.1537 & 0.0037 \\
$2007-9-17$ & -1.85 & -1.8490 & 0.001 \\
$2007-9-24$ & 1.40 & 1.4014 & 0.0014 \\
$2007-10-3$ & 1.30 & 1.2860 & -0.014 \\
$2007-10-11$ & 0.83 & 0.8312 & 0.0012 \\
$2008-3-2$ & 2.90 & 2.8898 & -0.0102 \\
$2008-3-5$ & 2.70 & 2.6833 & -0.0167 \\
\hline
\end{tabular}

Third section in different hole depth of measured Horizontal displacement value: 
TABLE 8 Random selected Third section Horizontal displacement data(mm)

\begin{tabular}{|c|c|c|c|c|c|c|}
\hline Date Elevation (m) & 137.8 & 139.3 & 141.3 & 142.8 & 144.3 & 146.3 \\
\hline $2006-7-2$ & 0.00 & 0.00 & 0.00 & 0.00 & 0.00 & 0.00 \\
$2006-9-4$ & -0.10 & 1.17 & 0.05 & 0.18 & -0.45 & -0.77 \\
$2006-9-10$ & -0.34 & 1.27 & -0.15 & -0.02 & -0.80 & -0.65 \\
$2006-9-18$ & -0.54 & 1.15 & -0.27 & -0.02 & -0.85 & -0.75 \\
$2006-9-23$ & -0.47 & 1.19 & -0.09 & -0.15 & -0.95 & -0.10 \\
$2006-9-27$ & -0.17 & 1.22 & -0.25 & -0.02 & -1.00 & -0.88 \\
$2006-10-2$ & -0.44 & 0.92 & -0.52 & -0.15 & -1.02 & -0.90 \\
$2006-10-7$ & -0.35 & 0.97 & -0.62 & -0.13 & -1.32 & -0.77 \\
$2006-10-12$ & -0.69 & 1.42 & -0.52 & -0.12 & -0.90 & -0.65 \\
$2006-10-16$ & -0.52 & 1.72 & -0.22 & -0.05 & -1.05 & -0.55 \\
$2006-11-5$ & -0.57 & 1.52 & -0.27 & -0.10 & -1.10 & -0.65 \\
$2007-8-3$ & 0.16 & -3.46 & 0.63 & 0.40 & 0.30 & -2.93 \\
$2007-8-13$ & -0.90 & 3.37 & -3.37 & -0.92 & -3.43 & 1.20 \\
$2007-8-24$ & 0.35 & -2.63 & 1.18 & 0.88 & 1.05 & -2.10 \\
$2007-8-31$ & 0.08 & -2.78 & 0.76 & 0.48 & 0.60 & -2.43 \\
$2007-9-10$ & -1.44 & -2.70 & 0.91 & 0.80 & 0.88 & -1.97 \\
$2007-9-19$ & 0.78 & -2.23 & 1.43 & 1.30 & 1.45 & -1.30 \\
$2007-9-28$ & 1.28 & -1.93 & 1.73 & 1.40 & 1.55 & -1.50 \\
$2007-10-5$ & 0.55 & -2.23 & 1.10 & 0.78 & 0.68 & -2.18 \\
$2007-10-13$ & 0.43 & -2.18 & 0.98 & 0.55 & 0.60 & -2.20 \\
$2008-3-2$ & -0.07 & -2.48 & 1.03 & 0.45 & 0.80 & -3.20 \\
$2008-3-5$ & -0.02 & -2.63 & 0.73 & 0.45 & 0.50 & -3.25 \\
\hline
\end{tabular}

1.Elevation $137.8 m$ :

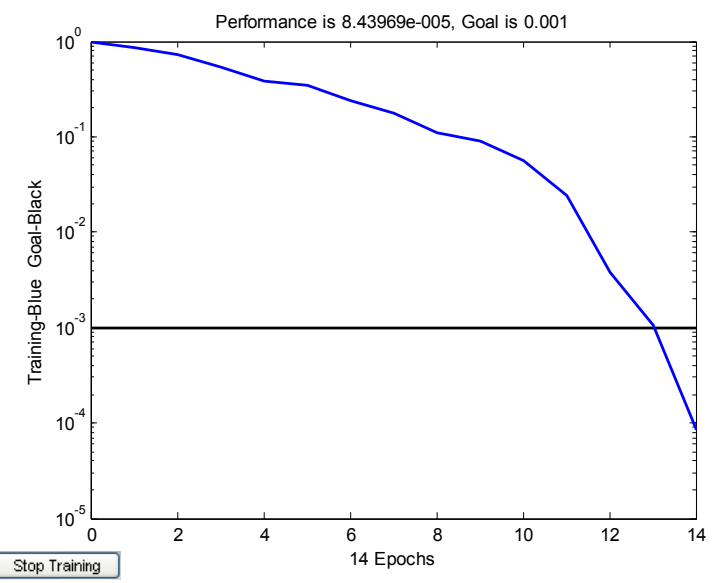

Fig. 19 training steps and performance

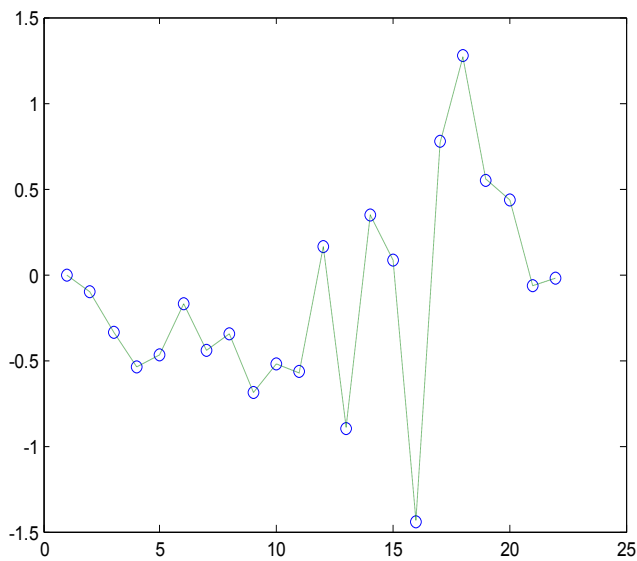

Fig.20 horizontal displacement data(Dotted line) prediction data(mark with circles)

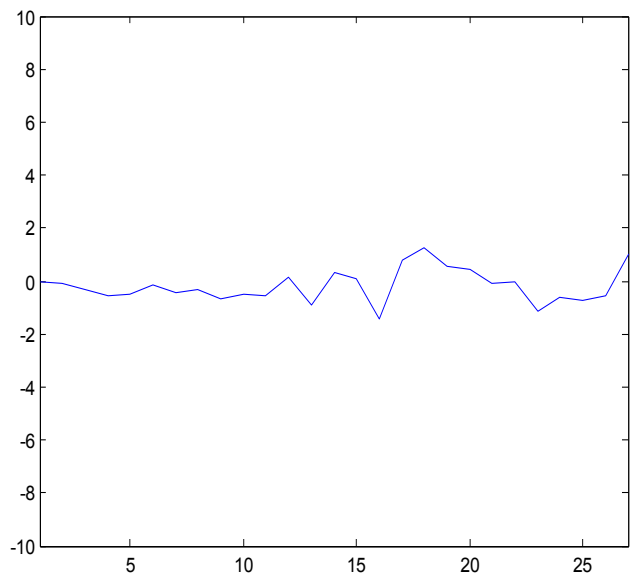

Fig.21 Elevation $137.8 \mathrm{~m}$ measured data and 5 steps prediction curve

\section{Elevation $139.3 m$ :}

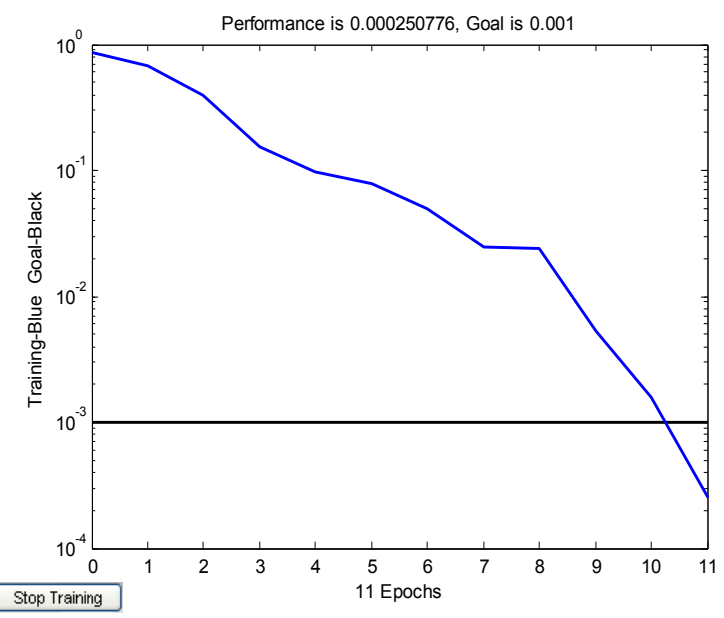

Fig.22 training steps and performance

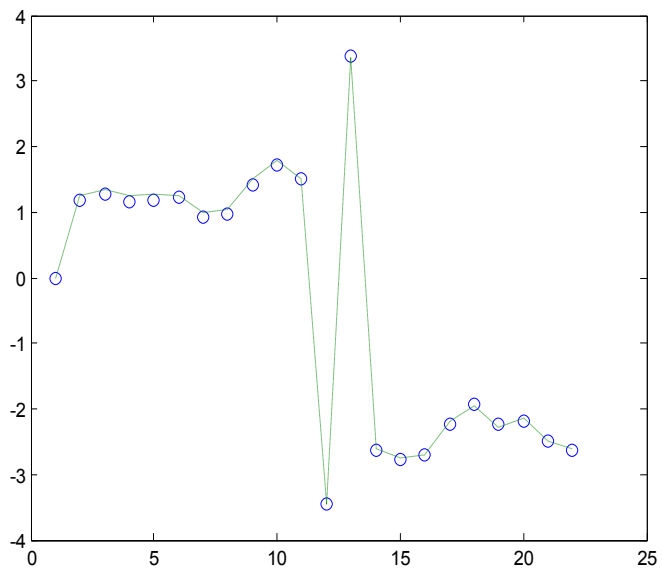

Fig.23 horizontal displacement data(Dotted line), prediction data(mark with circles) 


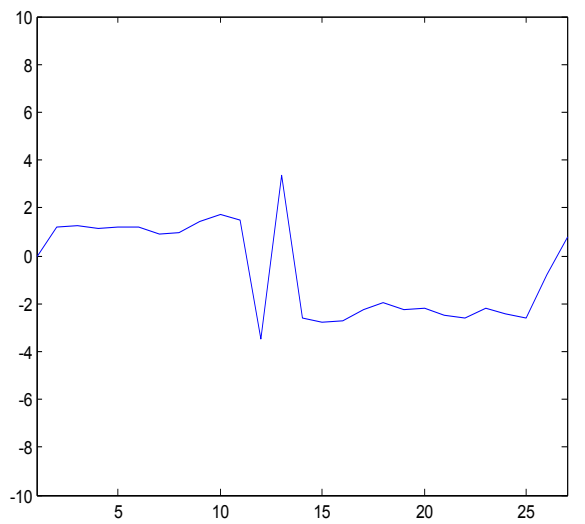

Fig.24 Elevation 139.3M measured data and 5 steps prediction curve

\section{Elevation $141.3 m$ :}

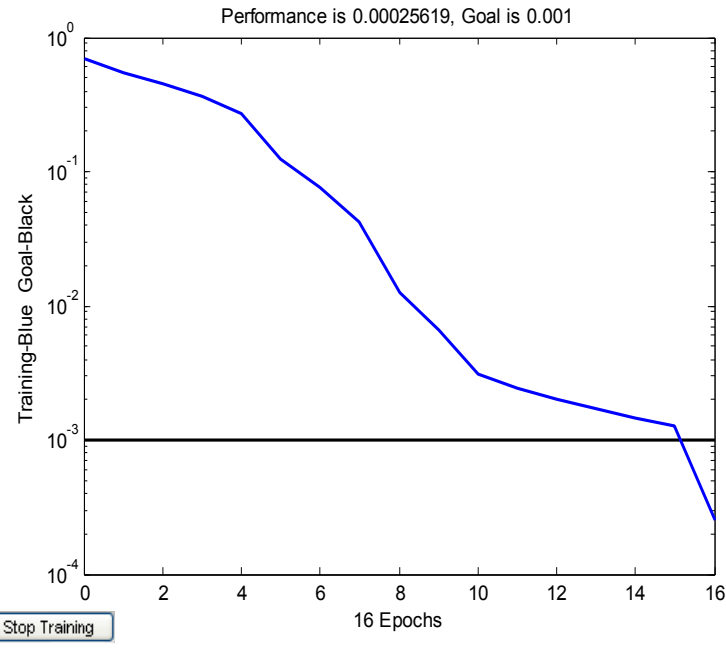

Fig.25 training steps and performance

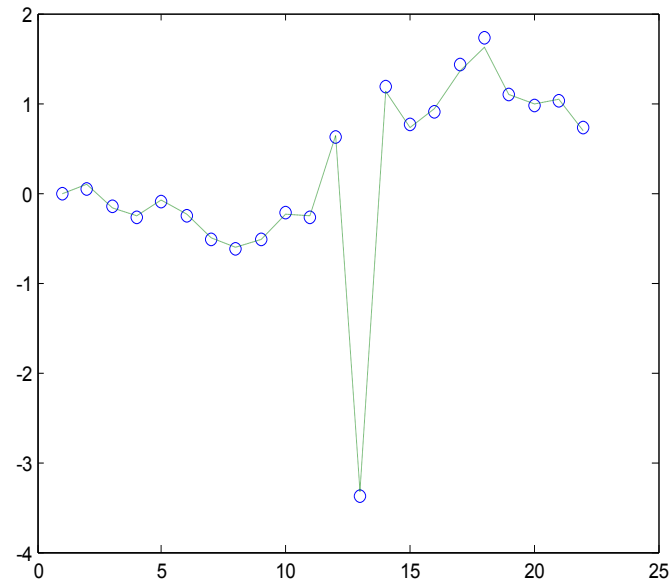

Fig.26 horizontal displacement data(Dotted line) prediction data(mark with circles)

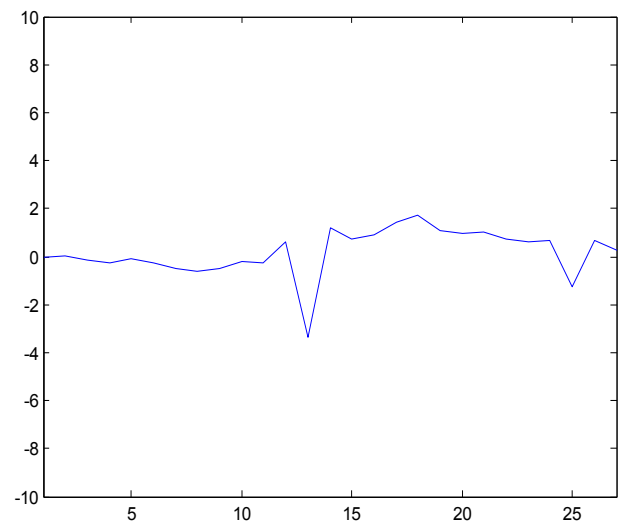

Fig.27 Elevation $141.3 \mathrm{~m}$ measured data and 5 steps prediction curve

\section{Elevation $142.8 m$ :}

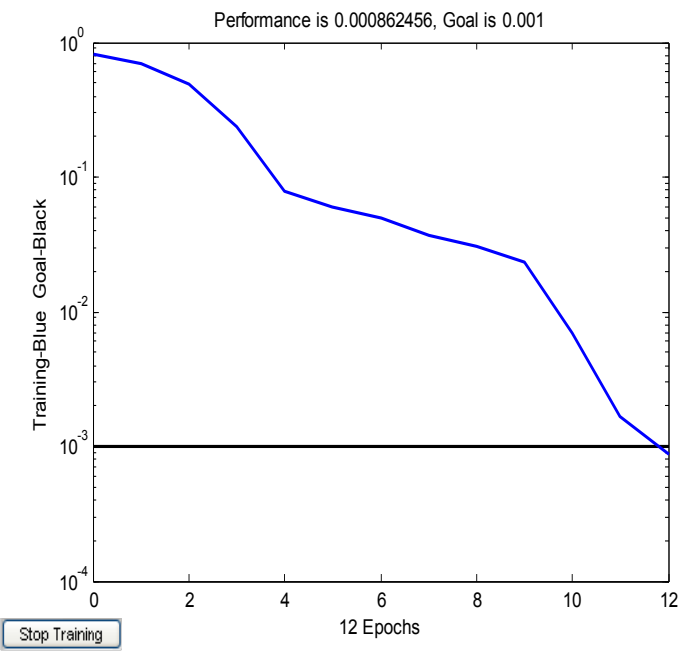

Fig.28 training steps and performance

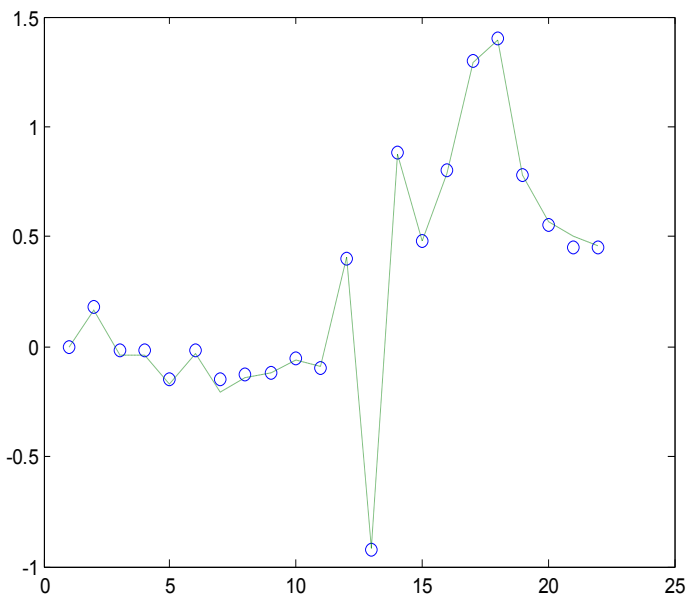

Fig.29 horizontal displacement data(Dotted line) prediction data(mark with circles) 


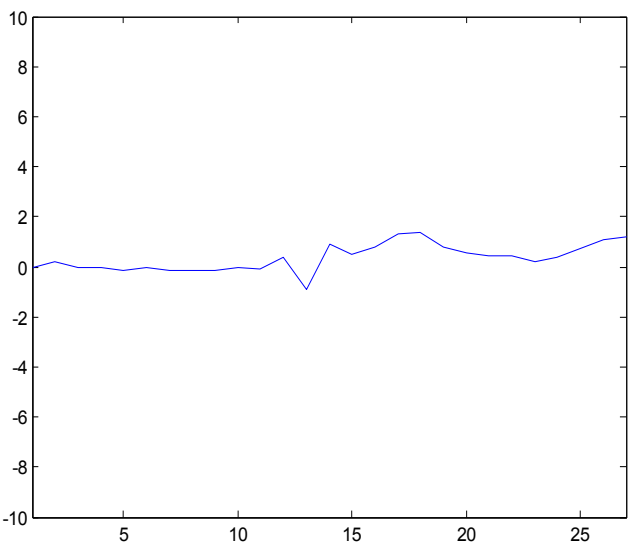

Fig.30 Elevation $142.8 \mathrm{~m}$ measured data and 5 steps prediction curve

\section{Elevation $144.3 m$ :}

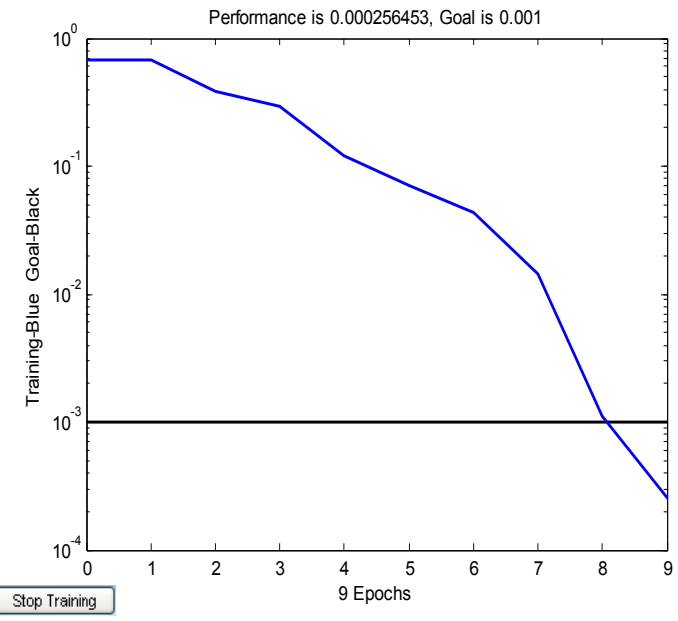

Fig.31 training steps and performance

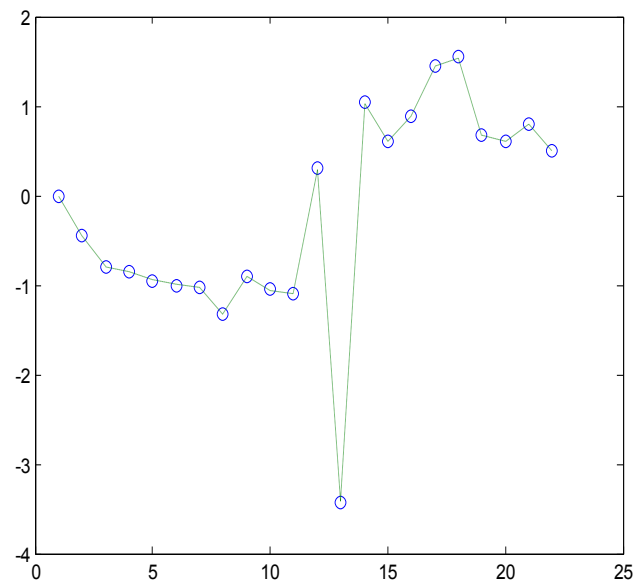

Fig.32 horizontal displacement data(Dotted line) prediction data(mark with circles)

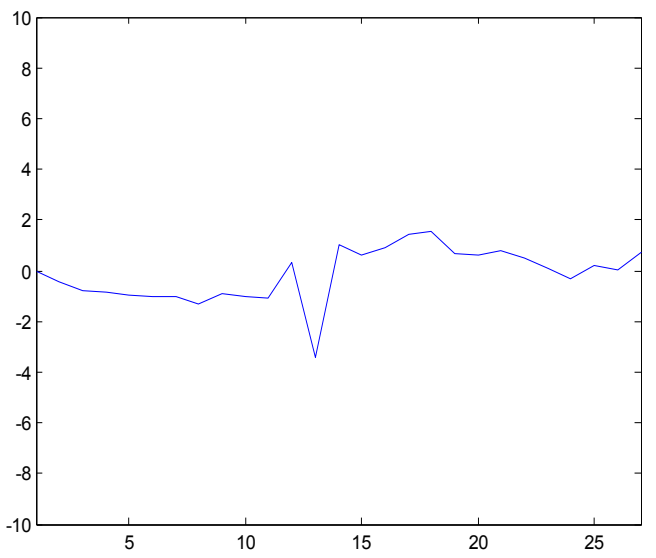

Fig.33 Elevation $144.3 \mathrm{~m}$ measured data and 5 steps prediction curve

\section{Elevation $146.3 m$ :}

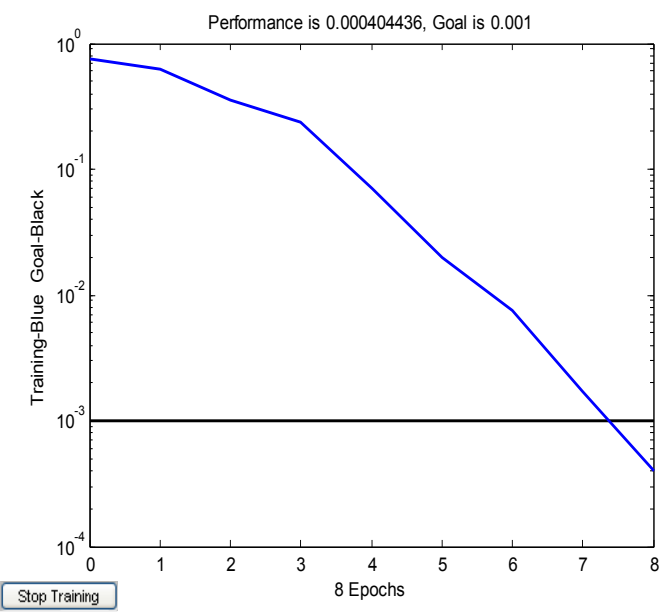

Fig.34 training steps and performance

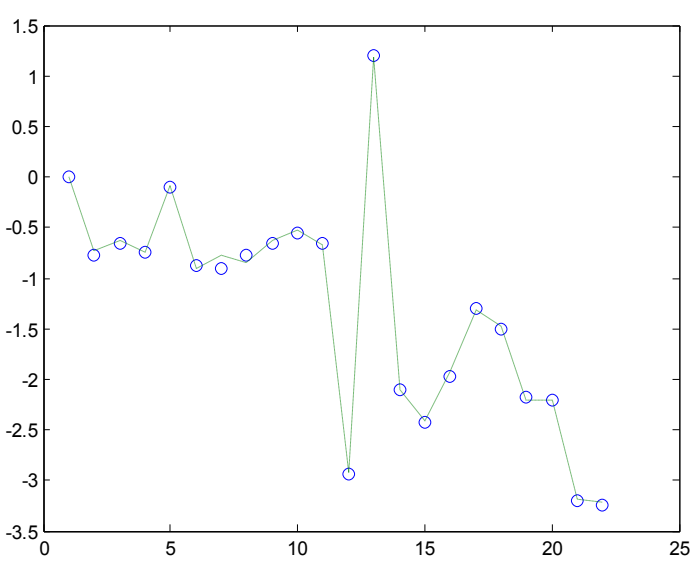

Fig. 35 horizontal displacement data(Dotted line) prediction data(mark with circles) 


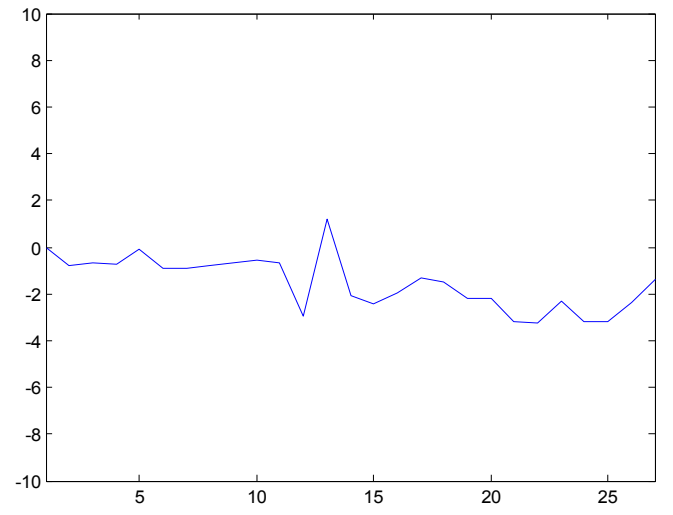

Fig.36 Elevation $146.3 \mathrm{~m}$ measured data and 5 steps prediction curve

From TABLE 2-7 and Fig.1-36,we can see prediction data nearly match the measured data, and prediction data show Breakwater project is stable and reliable.

\section{Conclusion}

By the analysis of relation of Measured data---frequency and stresses1 stresses2-----we can see the different parts have strong relevance, incidence relation Consistent and reliable. TABLE 9 are correlation coefficent matrix of frequency temperature stresses 1 stresses2, TABLE 10 are matrix of Covariance.
TABLE 9 correlation coefficent matrix

\begin{tabular}{|c|c|c|c|c|}
\hline & frequency & temperature & stresses1 & stresses2 \\
\hline frequency & 61930.77 & & & \\
\hline temperature & 24.86801 & 40.34717 & & \\
\hline stresses1 & 780.4391 & -0.01748 & 23.28016 & \\
\hline stresses2 & 941.9069 & -0.95002 & 24.7977 & 31.05626 \\
\hline
\end{tabular}

TABLE 10 matrix of Covariance

\begin{tabular}{|c|c|c|c|c|}
\hline & frequency & temperature & stresses1 & stresses2 \\
\hline frequency & 1 & & & \\
\hline temperature & 0.02161 & 1 & & \\
\hline stresses1 & 0.650067 & 0.001253 & 1 & \\
\hline stresses2 & 0.68008 & -0.02719 & 0.922379 & 1 \\
\hline
\end{tabular}

\section{References}

[1] S. Tamari *, J. H. M. Wösten and J. C. Ruiz-Suárez, Nov, 1996. Testing an Artificial Neural Network for Predicting Soil Hydraulic Conductivity. Soil Science Society of America Journal Vol. 60 No. 6, p. 1732-1741

[2] Sara Casciati. (2010) Response Surface Models to Detect and Localize Distributed Cracks in a Complex Continuum. Journal of Engineering Mechanics 136:9,p.1131-1142

[3] Bin Xu, Ping Lu, Yurong Guo, and Yan Xiao. 2010. Remote Structure Parameters Estimation on NetSlab Platform Using Response Time Series. p. $2477-2486$ 